International Journal of Information Sciences and Techniques (IJIST) Vol.3, No.4, July 2013

\title{
Assessing The Tangible And Intangible Impacts Of The Convergence Of E-Learning And Knowledge Management
}

\author{
Md. Sadiq Iqbal ${ }^{1}$, Md. Manirul Islam ${ }^{2}$ \\ ${ }^{1}$ Assistant Professor, Department of Computer Science \& Engineering, Bangladesh \\ University-Bangladesh \\ sadiq.iqbal abu . edu . bd \\ ${ }^{2}$ Assistant Professor, Department of Computer Science, American International \\ University-Bangladesh \\ manirul@aiub.edu
}

\begin{abstract}
Learning comes through creating and applying knowledge, whilst learning increases an individual's and organization's knowledge asset. Both e-learning and knowledge management feed off the same root: learning, improved capacity to perform work tasks, ability to make effective decisions, and positively impact the world around us. The difference between KM and e-learning is a function of time; knowledge management is dynamic, e-learning is static. As a medium, e-learning allows for the sharing of knowledge that has been tested, researched and organized. Knowledge management is much livelier. Conversations and sharing understanding happens in real time. Through KM, tacit understanding can be communicated, problems can be jointly solved, and serendipitous connections are formed. KM is chaotic, current. KM is ecology; e-learning is the architecture. E-learning courses become outdated, while KM environments are continually fresh and reflective of current activity in a field. Anyway, the strengths of the two fields need to be brought together. KM should feed into e-learning in order for the content of the "course" to remain fresh and to tap learners into a sustained knowledge environment after the course is done and e-learning should feed into the KM environment to provide easy mechanisms for organizing information in the manner that most of the people function. There"s no doubt that converging this two technology creates bigger impact in the learning process, but our discussion is focused to justify whether the convergence creates better value or not. In the light of the discussion, the conceptual link between these two key technologies has been drawn and several related issues are discussed.
\end{abstract}

\section{KEYWORDS}

Knowledge, E-learning, KM, EL, Convergence, BPR.

\section{INTRODUCTION}

According to Webster's Dictionary, knowledge is the fact or condition of knowing something with familiarity gained through experience or association. Knowledge may also be described as a set of models that describe various properties and behaviors within a domain. Knowledge may be recorded in an individual brain or stored in organizational processes, products, facilities, systems

DOI : $10.5121 /$ ijist.2013.3404 
and documents. For purposes of this report, we will focus upon the following definition of knowledge: The ideas or understanding which an entity possesses that is used to take effective action to achieve the entity's goal(s). This knowledge is specific to the entity that created it.

In today's fast paced society, an entity's knowledge base is quickly becoming its only sustainable competitive advantage. As such, this resource must be protected, cultivated and shared among its entity members. The full utilization of an entity's knowledge base, coupled with the potential of individual skills, competencies, thoughts, innovations and ideas will enable a company to compete more effectively in the future. With this, capturing and leveraging knowledge is an important new management trend. Coupled with this is the demand for better ways to develop better workplace learning strategies and performance improvement.

Periods of rapid change create a premium on learning - for both individuals and organizations. Prosperity and growth are the rewards of those who are the fastest at learning and putting their learning in action; stagnation and decline are the penalties for delay. In an era when it is knowledge rather than physical assets that increasingly defines competitive advantage, the process of managing knowledge becomes a central part of the learning process. Knowledge Management Defined "Knowledge management is an integrated systematic approach to identifying, managing and sharing all of an enterprise's information assets, including databases, documents, policies, and procedures, as well as previously unarticulated expertise and experience held by individual workers." Defining it further, Knowledge management refers to strategies and structures for maximizing the return on intellectual and information resources. Because intellectual capital resides both in tacit form (human education, experience and expertise) and explicit form (documents and data), KM depends on both cultural and technological processes of creation, collection, sharing, recombination and reuse. The goal is to create new value by improving the efficiency and effectiveness of individual and collaborative knowledge work while increasing innovation and sharpening decision-making.

"Knowledge Management caters to the critical issues of organizational adaptation, survival and competence in face of increasingly discontinuous environmental change. Essentially, it embodies organizational processes that seek synergistic combination of data and information processing capacity of information technologies and the creative and innovative capacity of human beings".

E-Learning Defined You may be familiar with terms such as distance learning, computer based training, online learning, or countless others. Many of these terms have overlapping meanings, and some experts may even disagree on the best term to use for a type of technology-assisted learning. We define e-learning as any form of learning that utilizes a network for delivery, interaction, or facilitation (in a few years you might not even use the computer). The network could be the Internet, a school or college LAN or even a corporate WAN. The learning could take place individually (guided or instructed by a computer) or as part of a class. Online classes meet either synchronously (at the same time) or asynchronously (at different times), or some combination of the two. E-learning combines communication, education, information, and training and is a core element of a successful e-business strategy. 
International Journal of Information Sciences and Techniques (IJIST) Vol.3, No.4, July 2013

\section{RELATIONSHIP BETWEEN E-LEARNING AND KNOWLEDGE MANAGEMENT}

\subsection{Similarities}

E-learning could be a cornerstone of knowledge management. True e-learning will foster a knowledge management process in which a "mini-economy" is created within the organization; the e-learning platform serves as an intermediary bringing those who need content with those who can provide it.

$\mathrm{KM}$ and e-learning share the same destiny. E-learning vendors are tuning to the training community's growing interest in KM practices and are positioning themselves accordingly. The term knowledge management appears with increasing frequency in product and service descriptions, and a small but growing number of firms are staking claims in the uncharted middle ground between the two disciplines. Established e-learning management vendors are also adding tools to encompass KM functions.

KM seeks to capture best practices passively but lacked the structure needed to succeed. Knowledge has to be captured in the way it's being communicated by the people who are doing the communication, and linking that knowledge to other content management tools that structure its delivery. This can be done through the synchronous e-learning tools or asynchronous forum.

\subsection{Differences}

Though there are similarities and common goal between KM and e-learning, there are important differences in practice. E-learning practices the longstanding traditional practice of assessment and testing to ensure skills and knowledge. This assessment is lacking in knowledge management practice since it focuses more on the free floating of ideas/knowledge. As E-learning moves from the classroom to the desktop and KM moves from theory to practice.

\subsection{Conceptual Link}

$\mathrm{KM}$ and organizational learning are increasingly similar in terms of tactics and tools they employ. Most of the learning benefits documented are attributable to KM practices, thus raising an important issue that if $\mathrm{KM}$ is a great way to get learning to happen, then training departments should be involved in harnessing KM. With this, we see the fusion among these three elements as seen in KM and in E-Learning:

\subsubsection{Content}

As e-learning developers work out ways to store and manage learning content in modular, objectbased formats, learning content could be served to users together with other knowledge resources from the same knowledge repository. The best of e-learning is built around or linked in directly to the actual knowledge repositories that are continually renewed and updated by the learning community. 
International Journal of Information Sciences and Techniques (IJIST) Vol.3, No.4, July 2013

\subsubsection{People}

In a mature e-learning and KM implementation, knowledge workers will tap the same knowledge well targeted for e-learning, unstructured information, in-house experts, and outside resources.

\subsubsection{Technology}

The technologies that leverage e-learning and knowledge management are beginning to overlap in a various ways. Technologies that enable such knowledge sharing as threaded discussion groups, chat rooms, synchronous meeting tools, and other collaborative software are among the technologies from KM field that are being adopted in e-learning settings.

Examples of these technologies are XML and learning object-based content design. But templatebased authoring tools that allow subject matter experts (SMEs) to put their knowledge into content format are also an important part of the combined e-learning and KM picture. Such tools, which use instructional design principles to guide users in authoring content, help free knowledge that is locked in reservoirs throughout an organization.

\section{MERGING OF E-LEARNING AND KM}

Knowledge management and e-learning are merging and leaving the traditional concept of classroom training behind. At least two factors are driving the fusion of knowledge management and e-learning:

Firstly, knowledge management and e-learning are essentially both about knowledge acquisition. The process of knowledge acquisition in the KM context is not much different from knowledge acquisition through self-paced interactive learning; the technology infrastructure employed is often the same and an equal investment of discipline and time is exerted by the employees in expanding their personal knowledge base and skills, whether they study from the knowledge management portal or the e-learning portal on the company intranet.

Secondly, both knowledge management and e-learning can encourage information sharing. Technology tools let employees contribute new pieces of knowledge in the context of workknowledge archived in a knowledge management repository--or in the context of learning--course chat discussions, for instance, which can generate new knowledge and then which is archived in the knowledge management repository.

Once information is captured and locked down as useful knowledge, its source becomes irrelevant in terms of its value.

\section{ISSUES OF CONVERGING KM AND EL}

Consensus is growing that the convergence of KM \& EL is a good thing, that it would continue and intensify, and that both fields can benefit from a greater partnership. Yet, there are lots of constraints that seem to be confronted at the crossroad of this convergence. 
International Journal of Information Sciences and Techniques (IJIST) Vol.3, No.4, July 2013

\subsection{Opportunities}

The key opportunities that the convergence will create follow:

\subsubsection{Business Process Re-Engineering}

The new business environment, characterized by dynamically discontinuous change, requires a re-conceptualization of knowledge management as it has been understood in information systems practice and research. One such conceptualization is proposed in the form of a sense-making model of knowledge management for new business environments. Application of this framework will facilitate business model innovation necessary for sustainable competitive advantage in the new business environment characterized by dynamic, discontinuous and radical pace of change."

\subsubsection{Knowledge Management and Re-Engineering}

While reengineering implies one-shot radical change in organizational processes to achieve maximum increases in efficiency, knowledge management implies continuous and ongoing renewal of organizational schemas to anticipate the future opportunities and threats. While reengineering shifts the organizational processes from one stage of mechanization to a more efficient phase of mechanization, knowledge management shifts the organization to an ongoing organic mode of functioning.

The basic premise of reengineering is embedded in 'fundamental rethinking' of the way of doing the business. However, such 'fundamental rethinking' is generally necessary if the theory of business has not encountered the 'reality check' of the business environment for an extended duration. Such fundamental transformations have caused drastic changes, often imposing such radical changes on the business processes and the humans involved in those processes. One may surmise that massive implementation failures of many reengineering efforts suggest that 'radical change' imposed upon the organizational processes and human elements don't necessarily ensure implementation success.

In contrast, knowledge management facilitates continuous and ongoing processes of learning and unlearning thus ensuring that need for imposing top-down 'radical change' may be minimized. In this view, it is recognized that change is the 'name of the game' unlike the electrical shock of reengineering that is needed to jumpstart the business processes. Furthermore, 'fundamental rethinking' doesn't get materialized in the form of top-down reshuffling of organizational processes, people and structures. It is ingrained in the day-to-day operations of the business at the grassroots level and driven by the people who interact with the external environment on the frontlines of the business. These are the people who are directly in touch with the dynamically changing reality of the business environment.

\subsection{Constraints}

The key constraints that would affect the convergence follow: 
International Journal of Information Sciences and Techniques (IJIST) Vol.3, No.4, July 2013

\subsubsection{Culture - Changing People's Behavior}

Whatever their formal plans, many companies that attempt to manage knowledge soon find themselves deep in the business of changing their cultures. Even in companies which know-how is a big deal, people don't share it readily or naturally. Employees aren't encouraged to talk about what they learn or even admit they need to learn something. Reading or browsing the Internet is often considered the behavior of slackers.

Larry Prusak of the IBM Consulting Group warns, "When it comes to successfully managing knowledge, culture trumps all other factors." Some organizations are fortunate to have had a knowledge-sharing culture before beginning to formalize their knowledge management. These firms, typically high-tech or knowledge-driven organizations, according to Davenport and Prusak in their book Working Knowledge, have the advantage that they already attract and hire employees who sought and applied knowledge while in school.

Even though there exists the concept of flat (network) structure of the organization especially in this age of information and knowledge, hierarchical structures are still being practiced in many organizations. May be due to this fact, each department are coming up with different idea of implementation about the same thing. This is a big hindrance in successful implementation of $\mathrm{KM}$ and e-learning in an organization. Hence, Awareness of the strategies/policies/goals implemented to the organization must be transparent to all in order to minimize the distance between higher level and lower level. The statement of Brooke Manville, chief learning officer for e-learning technology developer of Saba who was involved in the KM field's formative years as the first director of $\mathrm{KM}$ for management consultant McKinsey \& Company, is quite evident. "There was enough of difference between myself and my colleagues on what we wanted to do and KM was seen as another layer of complexity added to their already hectic jobs."

Since KM is merged with e-learning, the HRD must be fully knowledgeable and equipped with all the needed things to get content to the needed audience as quickly as possible. Top management must always realize the transformation or changes before it could expand to the least individual in the organization.

"To conceive of knowledge as a collection of information seems to rob the concept of all of its life...Knowledge resides in the user and not in the collection. It is how the user reacts to the collection of information that matters." - Churchman

\subsubsection{Measurement - Assessing the Value and Performance of Knowledge Assets}

One thing is certain: Managing knowledge is not a well-defined process. There are many questions that remain unanswered. What knowledge should you keep and what should you toss away? How do you capture the knowledge of chance hallway conversations? How do you make tacit knowledge explicit?

"Most extant knowledge management systems are constrained by their overly rational, static and a contextual view of knowledge. Effectiveness of such systems is constrained by the rapid and discontinuous change that characterizes new organizational environments. The prevailing knowledge management paradigm limits itself by its emphasis on convergence and consensusoriented processing of information. Strategy experts have underscored that the focus of 
International Journal of Information Sciences and Techniques (IJIST) Vol.3, No.4, July 2013

organizational knowledge management should shift from ,prediction of future ${ }^{e e}$ [that cannot be computed] to ,anticipation of surprise." Such systems may be enabled by leveraging the divergent interpretations of information based upon the meaning-making capability of human beings."- From Information Management to Knowledge Management: Beyond the 'Hi-Tech Hidebound' Systems.

KM must be output-oriented in determining the outcome of their training since it is very hard to assess. The employees must be given the chance to know technology. It must not be abrupt because it may result to phobia or ,backslide of productivity. It must be viewed as a support to their daily activities and not as another layer of complexity.

\subsubsection{Quality - Determining What Knowledge Should be Managed}

The New World of business imposes the need for variety and complexity of interpretations of information outputs generated by computer systems. Such variety is necessary for deciphering the multiple worldviews of the uncertain and unpredictable future. Any company that can figure out how to give its people the organizational knowledge they need -- at the point and time needed -can position itself to compete more effectively and succeed much faster. This is where e-learning comes. Many companies have vital knowledge resting with one individual and do little to make the knowledge more generally available. Many companies are unaware of their own knowledge base and evidence has shown that knowledge is often lost from a company through employee attrition or related cost saving measures. The enterprise that harnesses its intellectual capital can apply that asset to its business challenges and opportunities. Obtaining and using knowledge is an important new management trend. Knowledge management is serious, high-level business for companies in which innovative brainwork translates directly into products and profits. Managing your company's knowledge more effectively and exploiting it in the marketplace is the latest pursuit of those seeking competitive advantage. Knowledge and other forms of 'intellectual capital' are the 'hidden assets' in a company. They do not appear on the balance sheet in annual reports, yet they underpin value creation and future earnings potential. Knowledge intensive companies, like Microsoft and Sun Microsystems, have market values at least 10 times the value of their physical assets.

\subsubsection{Money - Justifying the Use of Scarce Resources for Knowledge Initiatives}

Technology costs money, lots of money. The up-front cost of purchase is just the tip of the iceberg. Either we have not been totally forthright in the way we have presented the full cost of ownership to our campus financial planners, or those who have made the investment decisions thought that they were, instead, making one-time purchases. Whatever the reason, we continue to have too many campus financial planners failing to deal with the reality of the financial commitment to the life cycle of ownership. This problem is equally severe whether we are dealing with equipment on the desktop or the costs of operating, maintaining and enhancing campus infrastructure. Expensive or not, value-added or not, technology is an indispensable element of teaching, research and administration on our campuses today. To pay for it planners and managers, at all levels, must engage in the unpalatable exercise of budget reallocation.

Because we have come to equate the outcome with the method of achieving it, some of our campuses have been paralyzed by the grip of fear that set in when funds began to dwindle. Technology has the potential to liberate campuses from the debilitating obsession with conserving scarce resources. 
International Journal of Information Sciences and Techniques (IJIST) Vol.3, No.4, July 2013

\subsubsection{Technological Limitations}

How do you avoid the perception that having the latest, best hardware or software equates to managing knowledge?

\subsubsection{Business and Technology Strategy Challenge of Next Generation KMS}

In the world of re-everything, automation of functions and rationalization of workflow, and redesign of business processes, most KM organizations need to develop adaptive capacity for redefining their business value propositions - that add greatest value to the business enterprise. Competitive survival and ongoing sustenance would depend on the ability to continuously redefine and adapt organizational goals, purposes, and the organization's "way of doing things". Greater technological integration will help in achieving more efficient optimization for knowledge harvesting.

\section{RECOMMENDATIONS}

- $\quad$ The issue of time always comes up first. Our greatest challenge is creating opportunities for people to spend time learning. One successful approach is to create formal learning networks so that the identification and transfer of effective practices becomes part of the job.

- We need to teach the skills for tacit learning and cultivate the roles that go with it. The secret is in the interaction: people with people, and people with information. Facilitate knowledge growth through culture and incentives.

- The whole company should be in the business of creating knowledge. Information is the input and insight is the output. The box in-between is knowledge creation. Knowledge infrastructure should be well defined and conspicuous.

- $\quad$ Organizations need to look at knowledge sharing beyond their own borders to encompass their extended enterprises, and emphasizes the ability of its system to provide self-service access to learning and management features.

\section{CONCLUSION}

Once Upon a Time...A teacher, on her way to the classroom, met a KM Researcher on his way to the lab. She said, "Hey hello, I don't believe I've ever seen you in the real world". He replied, "And I don't believe I've ever seen you except at the head of the class." They looked at each other and he said, "I didn't know teachers could be so informal, so flexible, so distributed." She said, "I didn't know KM researchers could be so dynamic, so practical, so collaborative." They made a date to meet. She rushed back to the classroom and put on her finest 3-layer infrastructure. He rushed back to the lab and put on his most dynamic categories. They rushed out to meet at "The Intersection of Infrastructure and Intranet" and discovered:

- $\quad$ They liked the real world

- $\quad$ They liked each other in the real world.

- $\quad$ They lived happily ever after. 
International Journal of Information Sciences and Techniques (IJIST) Vol.3, No.4, July 2013

\section{ACKNOWLEDGEMENT}

First of all we would like to thank Almighty God who gave us knowledge and strength to complete this paper. Also we would like to express our sincere appreciation and thanks to our friends, colleagues and our family for their spontaneous support and encouragement.

\section{REFERENCES}

\section{Book}

Daryl, M. et al, 2000. Knowledge Management: Classic and Contemporary Works. MIT Press, USA.

Nonaka, I. and Takeuchi, H, 1995. The Knowledge Creating Company. Oxford University Press, NY, USA

Marc J. R,, 2006. Beyond E-Learning: Approaches and Technologies to Enhance Organizational Knowledge, Learning, and Performance. John Wiley \& Sons Inc, San Francisco, CA

\section{Journal}

Andy, I., 2002. Success Factors for Development of Knowledge Management in e-Learning in Gulf Region Institutions. Journal of Knowledge Management Practice, ISSN: 1705-9232.

\section{Conference paper or contributed volume}

Byron, M. et al, 2003. Convergence of Knowledge management and E-learning: The GetSmart experience. Proceedings of the 3rd ACM/IEEE-CS joint conference on Digital Libraries, Washington, DC, USA, 135-146.

Frappaolo, C. and W. Toms, 1997. Knowledge Management: From Terra Incognito to Terra Firma, The Delhi Group, http://www.delphigroup.com/articles/1997/11071997KMThoughtMgmt.html Barron, T., 2000. A Smarter Frankenstein: The Merging of E-Learning and Knowledge Management, ASTD Learning Circuits, http://www.learningcircuits.org/aug2000/barron.html Allee, V, 2000. eLearning is Not Knowledge Management, http://www.linezine.com/2.1/ features/vaenkm.htm

Lenat, D., 1995. CYC: A Large-Scale Investment in Knowledge Infrastructure, Vol. 38 No. 11, Communications of the ACM.

World Wide Web Consortium Web Ontology Group, 2002. http://www.w3.org/2001/sw/WebOnt http://www.kmworld.com http://www.elearners.com http://www.brint.com 